\title{
Age, abdominal obesity, and glycated hemoglobin are associated with carotid atherosclerosis in type 2 diabetes patients with nonalcoholic fatty liver disease
}

\author{
Cristina Alina Silaghi 1,2, Horațiu Silaghi³, Anca Elena Crăciunn, ${ }^{4,5}$, Anca Fărcaș 5,6, \\ Horațiu Alexandru Colosi ${ }^{7}$, Daniel Tudor Cosma ${ }^{2,4}$, Raluca Paiss, Nicolae Hâncu ${ }^{5}$, \\ Carmen Emanuela Georgescu ${ }^{1}$
}

${ }^{1}$ Endocrinology Department, ,Iuliu Hatieganu” University of Medicine and Pharmacy, Cluj-Napoca, Romania, ${ }^{2}$ County Clinic Emergency Hospital, Cluj-Napoca, Romania, ${ }^{3} 5$ th Surgery Department, ,Iuliu Hatieganu” University of Medicine and Pharmacy, Cluj-Napoca, Romania, ${ }^{4}$ Diabet, Nutrition and Metabolic Diseases Department, ,Iuliu Hatieganu” University of Medicine and Pharmacy, Cluj-Napoca, Romania, ${ }^{5}$ Regina Maria Clinic, Cluj-Napoca, Romania, ${ }^{6} 1$ st Internal Medicine Department, „Iuliu Hatieganu” University of Medicine and Pharmacy, Cluj-Napoca, Romania, ${ }^{7}$ Medical Informatics and Biostatistics Department, ,Iuliu Hatieganu” University of Medicine and Pharmacy, Cluj-Napoca, Romania, ${ }^{8}$ Service Hépatogastroentérologie, Hôpital Pitié- Salpêtrière, Université Pierre et Marie Curie, INSERM UMRS 938, Paris, France.

\begin{abstract}
Aim: The aim of this study was to evaluate the impact of clinical parameters and indices of body composition on the relation between non-alcoholic fatty liver disease (NAFLD) and carotid intima-media thickness (cIMT), in a type 2 diabetes mellitus population (T2DM). Material and methods: We retrospectively enrolled 336 T2DM outpatients who regularly attended Regina Maria Clinic in Cluj. Clinical, anthropometric and biochemical parameters were measured. Ultrasonography (US) was used to assess hepatic steatosis (HS) in all patients and cIMT in 146 subjects. Body composition was assessed by bioelectric impedance (BIA, InBody 720) in all patients. Results: cIMT was correlated with age ( $\mathrm{r}=0.25 ; \mathrm{p}=0.004)$, systolic blood pressure $(\mathrm{r}=0.18 ; \mathrm{p}=0.041)$, glycated haemoglobin $\mathrm{A} 1 \mathrm{C}(\mathrm{HbA1C}, \mathrm{r}=0.20 ; \mathrm{p}=0.04)$, and with coronary artery disease $(\mathrm{r}=0.20 ; \mathrm{p}=0.007)$. HS did not correlate with cIMT $(r=0.04 ; p=0.64)$. cIMT was correlated with visceral fatty area $(V F A, r=0.18 ; p=0.014)$ but not with other indices of body composition. Homeostasis model assessment for insulin resistance (HOMA-IR) was not correlated with cIMT ( $\mathrm{r}=0.17 ; \mathrm{p}=0.086)$. After multivariate analysis, age, HbA1c, and VFA were good independent predictors of cIMT $(\mathrm{r}=0.45 ; \mathrm{p}<0.001)$. Conclusions: These results are suggestive that in T2DM patients, fatty liver is not a direct mediator of early carotid atherosclerosis. Our data indicate that visceral fat accumulation and HbA1C are determinant factors of cIMT sugesting that controlling abdominal obesity and hyperglicemia might reduce atherosclerotic disease risk in NAFLD-T2DM subjects.

Keywords: nonalcoholic fatty liver disease, carotid intima-media thickness, visceral adipose tissue, type 2 diabetes, glycated hemoglobin
\end{abstract}

\section{Introduction}

Nonalcoholic fatty liver disease (NAFLD) is a common and underdiagnosed chronic liver disease affect-

Received 13.05.2015 Accepted 25.06.2015

Med Ultrason

2015, Vol. 17, No 3, 300-307

Corresponding author: Horatiu Silaghi

5th Surgery Department, "Iuliu Hatieganu"

University of Medicine and Pharmacy Cluj-Napoca

11, Tabacarilor Street, 400139

Cluj-Napoca, România

Phone: +40745004930

E-mail: hsilaghi@yahoo.com ing up to one-third of the global population in Western countries [1]. The prevalence of NAFLD is increased up to $70 \%$ to $90 \%$ among type 2 diabetes mellitus (T2DM) patients $[2,3]$ and makes it an important public health problem. NAFLD spans a spectrum from nonalcoholic simple fatty liver to non-alcoholic steatohepatitis (NASH) and steatofibrosis with potential progression towards cirrhosis and hepatocellular carcinoma [4]. NAFLD is now considered a hepatic expression of metabolic syndrome (MetS) [5]. It is closely associated with abdominal obesity, atherogenic dyslipidemia, and diabetes, exposing subjects with NAFLD to an increased risk of developing cardiovascular disease (CVD). Many 
studies involving non-diabetic subjects have shown that NAFLD is independently associated with CVD $[6,7]$. Carotid intima-media thickness (cIMT) is a reliable index of subclinical atherosclerosis [8] and a mirror of atherosclerosis progression in NAFLD patients. Carotid IMT is higher for individuals with NASH than for those with simple steatosis and the histological severity of NAFLD independently predicts cIMT [9]. There is a close association between cIMT and abdominal fat distribution, viceral obesity being associated with accelerated progression of atherosclerosis and considered an important cardiovascular risk factor [10-12]. Targher et al demonstrated that in healthy, non-obese male subjects, the significant increase of cIMT in NAFLD subjects was largely mediated by increased visceral fat accumulation [13].

In T2DM subjects the link between fatty liver and atherosclerosis is less clear and different studies reported conflicting results. Many works showed no association between the degree of liver steatosis and early atherosclerosis as measured by US [14-16] or computed tomography [17] in T2DM patients. In contrast, other studies in T2DM subjects indicated a significant increase of cIMT in the presence of NAFLD that could be partially explained by the presence of insulin resistance $[18,19]$. Body fat distribution seems to play an important role in atherosclerosis progression in non-diabetic patients with NAFLD but in T2DM subjects this aspect is not completely known. The aim of this study was to evaluate whether there is an impact of clinical parameters and indices of body composition on the relation between NAFLD and cIMT, in a T2DM population.

\section{Material and methods}

\section{Patients and methods}

We retrospectively enrolled 363 T2DM outpatients who regularly attended diabetes evaluation at Regina Maria Clinic in Cluj, Romania, between 2013-2014. We excluded 27 patients with history of viral or other causes of chronic liver disease (autoimmune hepatitis, hemochromatosis, Wilson disease, alpha 1 antitrypsin deficiency) or alcohol consumption greater than $40 \mathrm{~g} /$ day. Finally 336 patients (127 females and 209 males) were considered for this study. Ultrasound (US) examination was used to assess hepatic steatosis in all subjects and cIMT in 146 patients. The study protocol was conformed to the ethical guidelines of the Declaration of Helsinki and was approved by the local Ethics Committee. Due to the retrospective design of the study the informed consent of the patients was not obtained.

\section{Clinical and laboratory assessment}

All 336 patients underwent a complete clinical and anthropometric evaluation, including: age, sex, weight, height, waist and hip circumference, systolic blood pressure (SBP) and diastolic blood pressure (DBP), alcohol consumption, previous medical history, and concomitant treatment. Body mass index (BMI) was calculated by dividing weight in kilograms by the square of height in meters. Overweight/obesity was diagnosed when BMI was $\geq 25.0 \mathrm{~kg} / \mathrm{m}^{2}$, according to the 1999 World Health Organisation criteria [20]. Blood pressure was measured at the right upper arm after patient had been seated quietly for at least 5 minutes. Hypertension was defined as SBP $\geq 140 \mathrm{mmHg}$ and/or DBP $\geq 85 \mathrm{mmHg}$, according to European Society of Hypertension (ESH), or current treatment for hypertension. We assessed blood samples for liver function tests, total cholesterol, HDL cholesterol, triglycerides level, blood creatinine level, fasting blood glucose, insulin levels, and glycated haemoglobin A1C (HbA1C). The upper limit for HbA1c for our laboratory was $5.8 \%$. Insulin resistance (IR) was determined by the homeostasis model assessment (HOMA-IR) and was defined as HOMA-IR $>2.7$ according to previous publications for European countries [21]. MetS was diagnosed by International Diabetes Federation 2009 criteria.

\section{Body fat measurements}

The precision body composition analysis was performed by bioelectric impedance analysis using a InBody 720 device (Biospace Co., South Korea) according to the recommendation provided in the user manual (INBODY720 User's Manual) [22]. Total body weight is the sum of body fat mass and fat free mass. Percent body fat (PBF-\%) indicates the percentage of body fat within the body weight. The standard PBF- $\%$ is $15 \%$ for men and $23 \%$ for women. Indices of body composition are measured in kilograms and also include soft lean mass representing fat free mass without mineral mass and skeletal muscle mass. InBody 720 device calculates visceral fat area representing the visceral adipose tissue content, with normal value under $100 \mathrm{~cm}^{2}$. The control of results' validity was done according to device specifications using the impedance values from the measurements at 6 frequencies displayed on the result sheet and invalid measurements were excluded from analysis [22].

\section{US measurements}

NAFLD was defined as the presence of hepatic steatosis on US. Hepatic US was performed in all patients after 12 hours fasting, by a single experienced radiologist by a high-resolution B-mode ultrasonography with a 5 MHz transducer (Philips HD11 XE ultrasound system). Each subject was examined in the supine and left lateral positions during quiet inspiration. Hepatic steatosis was 


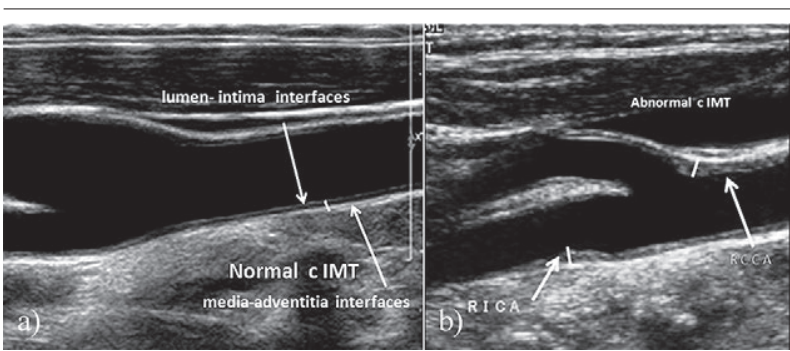

Fig 1. a) Normal cIMT measured as the distance between the lumen-intima and media-adventitia interfaces (indicated by white flashes) of the far wall of common carotid artery (CCA); b) Abnormal cIMT. cIMT thickness is marked by a white short line and the lumen-intima interface is indicated by a white flash.

diagnosed on the basis of characteristic US features: evidence of diffuse hyperechogenicity of the liver relative to the kidneys, ultrasound beam attenuation and poor visualization of intra-hepatic vessel borders and diaphragm. Semiquantitative US scoring for the degree of hepatic steatosis was not available in this study.

cIMT was measured by high-resolution real-time carotid B-mode ultrasonography with a 7.5-MHz linear transducer (Philips HD11 XE ultrasound system) bilaterally in longitudinal view at least $5 \mathrm{~mm}$ below the end of common carotid artery (CCA), the carotid bulb and the first $\mathrm{cm}$ of the internal carotid artery (ICA) always at a plaque-free point. cIMT was defined as the distance between the lumen-intima and media-adventitia interfaces of the far wall [23]. Two measurements from both sites were averaged to give the mean cIMT. A carotid plaque

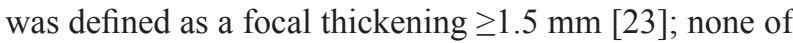
the study participants had clinically relevant carotid stenosis (i.e. $\geq 70 \%$ ) $[24,25]$. A cIMT value of more than 0.9 $\mathrm{mm}$ [26] or over the 75th percentile for the patient's age, sex, and race/ethnicity [27] was considered abnormal being associated with an increased risk of cardiovascular events $[28,29]$. Repeated measurements on the same subjects gave coefficients of variation $<5 \%$. A normal and an abnormal ultrasonographic aspect of cIMT are presented in figure 1.

\section{Statistic analysis}

Kolmogorov-Smirnov tests and quantile-quantile plots have been used to evaluate the distribution of all investigated quantitative variables. For normally distributed variables descriptive results were expressed as mean \pm standard deviation (SD), while non-normally distributed variables have been described in terms of median, minimum and maximum. Since most variables departed significantly from normal distribution, comparisons between groups based on the presence of hepatic steatosis, were performed using Mann-Whitney U tests for quantitative variables and Fisher's exact tests for categorical data. Correlations between quantitative variables were investigated using nonparametric Spearman correlation coefficients followed by multiple linear regression analysis to identify variables independently associated with cIMT. For further investigation regarding predictors of increased carotid wall thickness, cIMT has been dichotomized based on a $0.9 \mathrm{~mm}$ cut-off value [26,27], and multiple logistic regression analysis have been performed using iterative selection of covariates for optimal prediction of cIMT higher than $0.9 \mathrm{~mm}$. $\mathrm{P}<0.05$ was considered statistically significant. Statistical analysis were performed using IBM SPSS 21 statistical software (Chicago IL).

\section{Results}

Taking into consideration the results obtained by hepatic US, the 336 patients were divided in 2 groups: with hepatic steatosis $(86 \%)$ and without hepatic steatosis (14\%). Clinical and biochemical characteristics of the 2 groups are presented in Table I.

In 146 patients the results of both carotid and hepatic US were available. cIMT was correlated with age $(\mathrm{r}=0.25$; $\mathrm{p}=0.004)$, systolic blood pressure $(\mathrm{r}=0.18 ; \mathrm{p}=0.041)$, $\mathrm{HbA1C}(\mathrm{r}=0.20 ; \mathrm{p}=0.04)$, and with the presence of coronary artery disease $(r=0.20 ; p=0.007)$. Concerning body fat measurements, cIMT was correlated with visceral fat area $(\mathrm{r}=0.18 ; \mathrm{p}=0.014)$ but not with body fat mass, fat free mass, soft lean mass, skeletal muscle mass, or PBF-\%. There was no significant correlation between cIMT and fasting glucose $(\mathrm{r}=0.12 ; \mathrm{p}=0.087)$, HOMA-IR $(\mathrm{r}=0.17 ; \mathrm{p}=0.086)$, diabetes duration $(\mathrm{r}=0.6 ; \mathrm{p}=0.42)$ or presence of MetS $(r=0.04 ; p=0.58)$. No correlation was found between cIMT and total cholesterol, triglycerides, LDL and HDL cholesterol levels. cIMT was higher in patients with hepatosteatosis versus without hepatosteatosis but the difference was not significant $(\mathrm{p}=0.92)$. Hepatosteatosis did not correlate with cIMT $(\mathrm{r}=0.04 ; \mathrm{p}=0.64)$. After multivariate analysis, age, $\mathrm{HbAlc}$ and visceral fat area were good independent predictors of cIMT $(\mathrm{r}=0.45$; $\mathrm{p}<0.001)$. Table II presents two models of multiple regression analysis, with independent variables that contextually predicted the dependent variable cIMT.

After iterative selection of covariates for model optimization, our best logistic regression model included only age and sex as independent predictors of cIMT. Models also including NAFLD and visceral fat area respectively, still achieved reasonable prediction accuracy in the classification of cIMT according to the $0.9 \mathrm{~mm}$ cut-off value (table III). During the model optimization process, smoking, hypertension, statines, and other investigated predictors and possible confounders did not 
Table I. Clinical and biochemical characteristics of diabetic patients grouped according to the presence of hepatic steatosis as assessed by ultrasonography.

\begin{tabular}{|c|c|c|c|}
\hline & With hepatosteatosis & Without hepatosteatosis & $\mathbf{p}$ \\
\hline Number of subjects & 289 & 47 & \\
\hline Age (years) & $55.7 \pm 9.0$ & $59.6 \pm 9.5$ & 0.80 \\
\hline BMI (kg/m2) & $32.3(18.7,72.8)$ & $30.5(20.4,46.1)$ & $<0.0001$ \\
\hline $\mathrm{WC}(\mathrm{cm})$ & $112(78,162)$ & $102(74,149)$ & $<0.0001$ \\
\hline Diabetes duration (years) & $5(0,38)$ & $5(0,31)$ & 0.53 \\
\hline $\mathrm{SBP}(\mathrm{mmHg})$ & $145(100,212)$ & $141.5(92,230)$ & 0.56 \\
\hline DBP (mmHg) & $88(50,155)$ & $87(68,107)$ & 0.79 \\
\hline $\mathrm{TC}(\mathrm{mmol} / \mathrm{l})$ & $4.96(2.3,16.5)$ & $4.8(0.7,10.5)$ & 0.57 \\
\hline TGL (mmol/l) & $1.90(0.5,40.0)$ & $1.36(0.32,29.1)$ & 0.002 \\
\hline $\operatorname{HDLc}(\mathrm{mmol} / \mathrm{l})$ & $1.06(0.5,4.6)$ & $1.2(0.7,2.1)$ & 0.012 \\
\hline LDLc (mmol/l) & $2.73 \pm 1.57$ & $2.65 \pm 1.30$ & 0.65 \\
\hline TGL/HDLc & $4.0(0.6,269)$ & $2.8(0.6,99)$ & $<0.0001$ \\
\hline FPG (mmol/1) & $161(81,476)$ & $163(89,338)$ & 0.78 \\
\hline HOMA-IR & $5.6(0.83,25.9)$ & $3.9(1.14,9.35)$ & 0.004 \\
\hline $\mathrm{HbA1C}$ & $7.9(5,13)$ & $7.9(5,16)$ & 0.66 \\
\hline ASAT (units/l) & $24(10,191)$ & $21(10,60)$ & 0.011 \\
\hline ALAT (units/l) & $32(8,209)$ & $26(12,135)$ & 0.010 \\
\hline$\gamma \mathrm{GT}$ (units/l) & $39(13,947)$ & $32.5(12,112)$ & 0.025 \\
\hline BFM (kg) & $33.6(4.1,109.5)$ & $28.5(10.3,69.6)$ & $<0.0001$ \\
\hline VFA (cm2) & $172.19 \pm 48.86$ & $142.78 \pm 40.2$ & $<0.0001$ \\
\hline $\operatorname{cIMT}(\mathrm{mm})$ & $1.0(0.1,3)$ & $0.98(0.7,1.2)$ & 0.92 \\
\hline
\end{tabular}

Data are expressed as mean \pm standard deviation $(\mathrm{SD})$, or median (minimum, maximum). cIMT = carotid intima media thickness, $\mathrm{BMI}=$ body mass index, $\mathrm{WC}=$ waist circumference, $\mathrm{SBP}=$ systolic blood pressure, $\mathrm{DBP}=$ diastolic blood presure, $\mathrm{TC}=$ total cholesterol, $\mathrm{TGL}=$ triglycerides, $\mathrm{HDLc}=$ high density lipoprotein, $\mathrm{LDLc}=$ low density lipoprotein, $\mathrm{FPG}=$ fasting plasma glucose, $\mathrm{HbA1C}=$ glycated haemoglobin, HOMA-IR = homeostasis model assessment insulin resistance, ALAT $=$ alanine aminotransferase, ASAT $=$ aspartate aminotransferase, $\gamma \mathrm{GT}=\gamma$ glutamyl transferase, MetS $=$ metabolic syndrome, $\mathrm{BFM}=$ body fat mass, VFA $=$ visceral fat area.

Table II. Multiple linear regression analysis. Models 1 and 2: Age, HbA1C, HOMA-IR and VFA were significant independent predictors of cIMT.

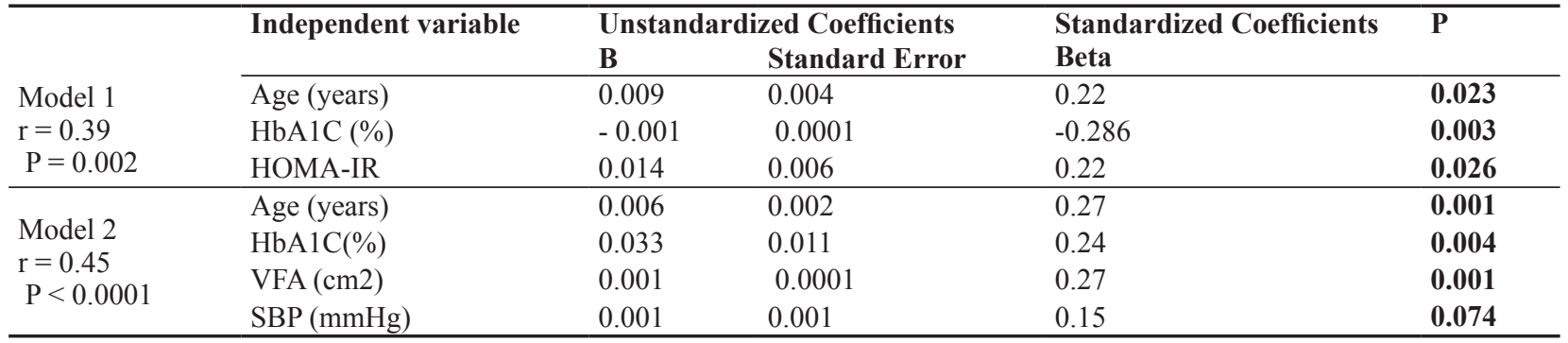

$\mathrm{SBP}=$ systolic blood pressure, $\mathrm{HbA1C}=$ glycated haemoglobin, HOMA-IR $=$ homeostasis model assessment insulin resistance, $\mathrm{VFA}=$ visceral fat area, $\mathrm{cIMT}=$ carotid intima media thickness.

Table III. Multiple logistic regression analysis: age and sex were significant predictors of cIMT higher than $0.9 \mathrm{~mm}$.

\begin{tabular}{|c|c|c|c|c|c|c|c|c|}
\hline \multirow{2}{*}{ Model } & \multirow{2}{*}{ Prediction accuracy } & \multirow{2}{*}{ Independent variable } & \multirow{2}{*}{ B } & \multirow{2}{*}{ SE } & \multirow[b]{2}{*}{$\mathbf{p}$} & \multirow{2}{*}{ Odds Ratio (OR) } & \multicolumn{2}{|c|}{ 95\% CI for OR } \\
\hline & & & & & & & Lower & Upper \\
\hline \multirow[t]{2}{*}{ Model 1} & $75.7 \%$ & Age (years) & 0.113 & 0.028 & 0.000 & 1.120 & 1.061 & 1.182 \\
\hline & & Sex (male/female) & 1.090 & 0.441 & 0.013 & 2.975 & 1.253 & 7.061 \\
\hline \multirow[t]{3}{*}{ Model 2} & $75.3 \%$ & NAFLD (yes/no) & -0.429 & 0.600 & 0.475 & 0.651 & 0.201 & 2.111 \\
\hline & & Age (years) & 0.114 & 0.028 & 0.000 & 1.120 & 1.061 & 1.183 \\
\hline & & Sex (male/female) & 1.118 & 0.444 & 0.012 & 3.058 & 1.281 & 7.301 \\
\hline \multirow[t]{3}{*}{ Model 3} & $75.3 \%$ & $\mathrm{VFA}(\mathrm{cm} 2)$ & 0.005 & 0.004 & 0.195 & 1.005 & 0.997 & 1.013 \\
\hline & & Age (years) & 0.113 & 0.028 & 0.000 & 1.120 & 1.060 & 1.183 \\
\hline & & Sex (male/female) & 0.992 & 0.450 & 0.027 & 2.697 & 1.117 & 6.510 \\
\hline
\end{tabular}

$\mathrm{VFA}=$ visceral fat area, NAFLD $=$ non-alcoholic fatty liver disease, $\mathrm{cIMT}=$ carotid intima media thickness. 
significantly contribute to the prediction of a cIMT higher than $0.9 \mathrm{~mm}$.

\section{Discussions}

Our results indicate that hepatic steatosis was present in $86 \%$ of T2DM subjects. The prevalence of hepatic steatosis assessed by US was slightly greater than previous reports proving that NAFLD is very common in subjects with T2DM $[2,3,6,15]$. Our T2DM patients with hepatic steatosis presented a cluster of cardiovascular risk factors with increased values of triglycerides and decreased level of HDL cholesterol, results consistent with previous evidences $[14,15,30]$. However, Targher et al showed that NAFLD was associated with an increased incidence of CVD in T2DM patients, independent of traditional CVD risk factors and MetS components [6]. They also found an increased incidence of atrial fibrillation in NAFLD-T2DM patients independently of important clinical risk factors for atrial fibrillation [31].

We did not find a correlation between hepatic steatosis and carotid atherosclerosis evaluated by cIMT. Nevertheless, body fat mass and measures of central obesity like waist circumference and visceral fat area were significantly linked to hepatic steatosis. This finding is in agreement with other works in T2DM subjects [14-16] showing no association between NAFLD and early atherosclerosis as measured by cIMT. In T2DM, the link between NAFLD and cIMT rests debatable. Among diet-controlled T2DM individuals the significant increase of cIMT in the presence of NAFLD is largely explained by HOMA-estimated IR [18]. Recently, Kim et al concluded that NAFLD not accompanied by insulin resistence is not associated with a carotid atherosclerotic burden [19]. We could not demonstrate a correlation between cIMT and HOMA-IR in our T2DM patients. The impact of HOMA-IR on the relation between cIMT and NAFLD could not be analyzed because of missing data and unequal groups of subjects to compare. Contraversely with T2DM, in healthy, non-obese male subjects, increase of cIMT in NAFLD subjects was related to abdominal accumulation [13], data supporting the potential adverse impact of MetS parameters on the relationship between NAFLD and carotid atherosclerosis. Other studies suggested a direct impact of NAFLD on carotid atherosclerosis throughout hepatic inflammation characteristic of NAFLD that could be an important contributor to systemic inflammation and accelerated atherogenesis [32]. McKimmnie at al observed a strong correlation between pro-atherogenic biomarkers and the elements of the MetS suggesting that hepatic steatosis reflects more than general adiposity, and represents a systemic, inflammatory, pro-atherogenic adipose state [17].

Moreover, we found that visceral fat area is correlated with cIMT in our T2DM study population. In the presence of BMI indicating obesity, the abdominal accumulation of adipose tissue seems to play a crucial role in atherosclerosis progression. We observed that other indices of body fat measurement and BMI were not correlated with cIMT, suggesting the importance of adipose tissue distribution. However, after adjustment for BMI and waist circumference, liver fat was not significantly associated with sub-clinical atherosclerosis [33]. Consistent with our findings, previous studies reported a significant link between abdominal fat distribution and cIMT in adults $[10,11]$ or obese adolescents. Visceral fat was a key predictor of arterial wall thickening, suggesting that the focus of cardiovascular disease prevention should be visceral obesity, and not blood lipids or lipid subclasses [34]. Visceral fat could be considered a mediator between NAFLD and cIMT through its secretion of multiples proinflammatory and pro-atherosclerotic factors [13]. Additionally, we found that visceral fat area is an indepedent predictor of cIMT, emphasizing the emerging concept of central obesity in the progression of atherosclerosis. This result is also validated by recent studies in diabetic [35] and non-diabetic patients of different races [12]. Visceral adipose tissue volume measured by US may be a better predictor of subclinical carotid atherosclerosis than waist circumference in healthy individuals [36].

The presence of carotid plaque or cIMT greater than $0.9 \mathrm{~mm}$ [26], or equal to 75th percentile for the patient's age, sex, and race/ethnicity are indicative of increased CVD risk [27]. For our group of T2DM patients we found that only age and sex significantly contribute to the prediction of a cIMT higher than $0.9 \mathrm{~mm}$. Hypertension/ongoing treatment, statines, smoking, visceral fat area or NAFLD are not independent predictors of cIMT higher than 0.9 $\mathrm{mm}$. The peculiarities of our T2DM population are the very high overall cIMT suggesting an increased risk of CVD for these subjects and an atherogenic dislipidemia profile in the patients with steatosis, who were more insulin resistant and had a higher level of transaminases.

In obese children and adolescents without diabetes, HbA1C was associated with increased cIMT independently of traditional CVD risk factors [37]. We found that $\mathrm{HbA1C}$ is an independent predictor of cIMT indicating that in T2DM patients with NAFLD we should give more attention to carotid atherosclerosis, especially in those with hyperglycemia. Interestingly, NAFLD was independently associated with coronary artery disease only in T2DM patients with higher $\mathrm{HbAlc}$, after adjustment for confounders [38]. 
This study has some limitations that should be mentioned. Firstly, the retrospective design of the study could introduce a bias concerning the selection of patients for abdominal and vascular ultrasonography. Secondly, US was used to measure cIMT in 146 of 336 T2DM subjects. However, the number of patients included in the study ensured an adequate sample size for multivariate analysis. Thirdly, liver biopsies were not available in our subjects. Liver biopsy is invasive and may result in severe complications but is considered the gold standard technique for identifying NAFLD. Other methods such as proton magnetic resonance spectroscopy and magnetic resonance imaging demonstrate high accuracy in the quantification of liver fat, but are limited by low availability, high cost and lack of standardization [39,40]. We used US to identify hepatic steatosis, a method that allows the detection of steatosis only when hepatocytes fat accumulation is above $15-20 \%$ [41,42]. Studies confirmed by biopsies indicate that the increased parenchymal echogenicity has a positive predictive value of 0.87 to 0.94 for steatosis diagnosis, according to the used criteria $[41,43]$. US has $60-94 \%$ sensitivity and $88-95 \%$ specificity in detecting hepatic steatosis [41,44], with $80 \%$ sensitivity at fat accumulation above $30 \%$ and reduced sensitivity when hepatic fat infiltration on biopsy is under 33\% [45]. The downsides of hepatic US include its inaccuracy in differentiating fibrosis from steatosis, in reproducibility and in the exact quantification of fat accumulation [41]. Fourthly, we could not completely exclude secondary causes of liver disease, even if subjects with a history of chronic liver disease or alcohol consumption were excluded from the study. This limitation could have increased the number of included patients and may explain the greater prevalence of NAFLD in this study compared to other studies in T2DM patients $[2,3]$. Finally, we did not directly measure abdominal visceral fat by L4-L5 computed tomography, the gold standard for measuring visceral fat area. In this study we used bioelectric impedance analysis, an easily applicable and useful method for evaluating body fat distribution [46]. Prior studies established bioelectric impedance analysis as an objective indicator of body composition and validated the measurement of visceral fat area by this method [47]. The visceral fat area obtained by bioelectric impedance analysis was correlated with the total and visceral fat in L4 measured by computed tomography in normal and HIV-positive patients [48].

The results of this study strongly support a constitutive role of visceral fat in the development of atherosclerosis and highlight the increased CVD risk in abdominal obese T2DM patients with NAFLD. Additionally, interventional studies reported a beneficial effect of weight loss, known to primarily reduce intra-abdominal fat depots, on the progression rate of early carotid atherosclerosis in obese individuals [49,50]. In patients with T2DM, the control of abdominal obesity appears to have a significant influence on the progression of subclinical atherosclerosis. Subsequently, an important concern is to decrease visceral obesity in order to reduce the frequency of atherosclerotic diseases. The identification of NAFLD abdominal obese T2DM patients could help in CVD risk prediction, with important management implications and benefit from early risk-reduction intervention.

\section{Conclusions}

The results of our study are suggestive for the fact that in T2DM patients, fatty liver seems not to be a direct mediator of early carotid atherosclerosis. Our data underline the importance of visceral fat accumulation and glycated hemoglobin for cIMT, suggesting that controlling abdominal obesity and hyperglicemia might reduce atherosclerotic disease risk in NAFLD-T2DM subjects.

Acknowledgement: This paper was published under the frame of the European Social Found, Human Resources Development Operational Programme 20072013, project no. POSDRU/159/1.5/S/138776.

\section{Conflict of interest: none}

\section{References}

1. Tilg H, Kaser A. Treatment strategies in nonalcoholic fatty liver disease. Nat Clin Pract Gastroenterol Hepatol 2005; 2: $148-155$.

2. Williamson RM, Price JF, Glancy S, et al. Edinburgh Type 2 Diabetes Study Investigators.Prevalence of and risk factors for hepatic steatosis and nonalcoholic fatty liver disease in people with type 2 diabetes: the Edinburgh Type 2 Diabetes Study. Diabetes Care 2011; 34: 1139-1144.

3. Gupte P, Amarapurkar D, Agal S, et al. Non-alcoholic steatohepatitis in type 2 diabetes mellitus. J Gastroenterol Hepatol 2004; 19: 854-858.

4. Pais R, Charlotte F, Fedchuk L, et al. LIDO Study Group.A systematic review of follow-up biopsies reveals disease progression in patients with non-alcoholic fatty liver. J Hepatol 2013; 59: 550-556.

5. Marchesini G, Bugianesi E, Forlani G, et al. Nonalcoholic fatty liver, steatohepatitis, and the metabolic syndrome. Hepatology 2003; 37: 917-923.

6. Targher G, Bertolini L, Rodella S, et al. Nonalcoholic fatty liver disease is independently associated with an increased incidence of cardiovascular events in type 2 diabetic patients. Diabetes Care 2007; 30: 2119-2121. 
7. Sookoian S, Pirola CJ. Non-alcoholic fatty liver disease is strongly associated with carotid atherosclerosis: a systematic review. J Hepatol 2008; 49: 600-607.

8. O'Leary DH, Polak JF. Intima-media thickness: a tool for atherosclerosis imaging and event prediction. Am J Cardiol 2002; 90: 18L-21L.

9. Targher G, Bertolini L, Padovani R, et al. Relations between carotid artery wall thickness and liver histology in subjects with nonalcoholic fatty liver disease. Diabetes Care 2006; 29: $1325-1330$.

10. Lakka TA, Lakka HM, Salonen R, Kaplan GA, Salonen JT. Abdominal obesity is associated with accelerated progression of carotid atherosclerosis in men. Atherosclerosis 2001; 154: 497-504.

11. De Michele M, Panico S, Iannuzzi A, et al. Association of obesity and central fat distribution with carotid artery wall thickening in middle-aged women. Stroke 2002; 33: 2923 2928.

12. Oike M, Yokokawa H, Fukuda H, et al. Association between abdominal fat distribution and atherosclerotic changes in the carotid artery. Obes Res Clin Pract 2014; 8: e448-e458.

13. Targher G, Bertolini L, Padovani R, Zenari L, Zoppini G, Falezza G. Relation of nonalcoholic hepatic steatosis to early carotid atherosclerosis in healthy men: role of visceral fat accumulation. Diabetes Care 2004; 27: 2498-2500.

14. Petit JM, Guiu B, Terriat B, et al. Nonalcoholic fatty liver is not associated with carotid intima-media thickness in type 2 diabetic patients. J Clin Endocrinol Metab 2009; 94: 41034106.

15. Poanta LI, Albu A, Fodor D. Association between fatty liver disease and carotid atherosclerosis in patients with uncomplicated type 2 diabetes mellitus. Med Ultrason 2011; 13: 215-219.

16. Coracina A, Gaiani S, Cosma A, et al. No association between the degree of liver steatosis and early signs of vasculopathy in T2DM. Nutr Metab Cardiovasc Dis 2012; 22: e11-e12.

17. McKimmie RL, Daniel KR, Carr JJ, et al. Hepatic steatosis and subclinical cardiovascular disease in a cohort enriched for type 2 diabetes: the Diabetes Heart Study. Am J Gastroenterol 2008; 103: 3029-3035.

18. Targher G, Bertolini L, Padovani R, et al. Non-alcoholic fatty liver disease is associated with carotid artery wall thickness in diet-controlled type 2 diabetic patients. J Endocrinol Invest 2006; 29: 55-60.

19. Kim SK, Choi YJ, Huh BW, et al. Nonalcoholic Fatty liver disease is associated with increased carotid intimamedia thickness only in type 2 diabetic subjects with insulin resistance. J Clin Endocrinol Metab 2014; 99: 1879-1884.

20. Obesity: preventing and managing the global epidemic. Report of a WHO consultation. World Health Organ Tech Rep Ser 2000; 894: 1-253.

21. Bugianesi E, Pagotto U, Manini R, et al. Plasma adiponectin in nonalcoholic fatty liver is related to hepatic insulin resistance and hepatic fat content, not to liver disease severity. J Clin Endocrinol Metab 2005; 90: 3498-3504.
22. INBODY 720 USER'S MANUAL. (1996-2004 Biospace Co, Ltd. URL http://www.bodyanalyse.no/docs/720\%20 users\%20manual.pdf.

23. Touboul PJ, Hennerici MG, Meairs S, et al. Mannheim Carotid Intima-Media Thickness and Plaque Consensus (2004-2006-2011). Cerebrovasc Dis 2012; 34: 290-296.

24. Staikov IN, Nedeltchev K, Arnold M, et al. Duplex sonographic criteria for measuring carotid stenoses. J Clin Ultrasound 2002; 30: 275-281.

25. Moneta GL, Edwards JM, Papanicolaou G, et al. Screening for asymptomatic internal carotid artery stenosis: duplex criteria for discriminating $60 \%$ to $99 \%$ stenosis. J Vasc Surg 1995; 21: 989-994.

26. Mancia G, Fagard R, Narkiewicz K, et al. ESH/ESC Guidelines for the management of arterial hypertension: the Task Force for the management of arterial hypertension of the European Society of Hypertension (ESH) and of the European Society of Cardiology (ESC). J Hypertens 2013; 31: 1281-1357.

27. Stein JH, Korcarz CE, Hurst RT, et al; American Society of Echocardiography Carotid Intima-Media Thickness Task Force. Use of carotid ultrasound to identify subclinical vascular disease and evaluate cardiovascular disease risk: a consensus statement from the American Society of Echocardiography Carotid Intima-Media Thickness Task Force. Endorsed by the Society for Vascular Medicine. J Am Soc Ecocardiogr 2008; 21: 93-111.

28. Lorenz MW, von Kegler S, Steinmetz H, Markus HS, Sitzer M. Carotid intima-media thickening indicates a higher vascular risk across a wide age range: prospective data from the Carotid Atherosclerosis Progression Study (CAPS). Stroke 2006; 37: 87-92.

29. Lorenz MW, Markus HS, Bots ML, Rosvall M, Sitzer M. Prediction of clinical cardiovascular events with carotid intima-media thickness. a systematic review and metaanalysis. Circulation 2007; 115: 459-467.

30. Toledo FG, Sniderman AD, Kelley DE. Influence of hepatic steatosis (fatty liver) on severity and composition of dyslipidemia in type 2 diabetes. Diabetes Care 2006; 29: 1845-1850.

31. Targher G, Valbusa F, Bonapace S, et al. Non-alcoholic fatty liver disease is associated with an increased incidence of atrial fibrillation in patients with type 2 diabetes. PLoS One 2013; 8: e57183.

32. Brea A, Mosquera D, Martin E, Arizti A, Cordero JL, Ros E. Nonalcoholic fatty liver disease is associated with carotid atherosclerosis: a case- control study. Arterioscler Thromb Vasc Biol 2005; 25: 1045-1050.

33. Dick TJ, Lesser IA, Leipsic JA, Mancini GB, Lear SA. The effect of obesity on the association between liver fat and carotid atherosclerosis in a multi-ethnic cohort. Atherosclerosis 2013; 226: 208-213.

34. Slyper AH, Rosenberg H, Kabra A, et al. Early atherogenesis and visceral fat in obese adolescents. Int J Obes (Lond) 2014; 38: 954-958.

35. Bacha F, Edmundowicz D, Sutton-Tyrell K, Lee S, Tfayli $\mathrm{H}$, Arslanian SA. Coronary artery calcification in obese 
youth: what are the phenotypic and metabolic determinants? Diabetes Care 2014; 37: 2632-2639.

36. Rallidis LS, Baroutsi K, Zolindaki M, et al. Visceral adipose tissue is a better predictor of subclinical carotid atherosclerosis compared with waist circumference. Ultrasound Med Biol 2014; 40: 1083-1088.

37. Chen LH, Zhu WF, Liang L, et al. Relationship between glycated haemoglobin and subclinical atherosclerosis in obese children and adolescents. Arch Dis Child 2014; 99: 39-45.

38. Kwak MS, Yim JY, Kim D, et al. Nonalcoholic fatty liver disease is associated with coronary artery calcium score in diabetes patients with higher HbA1c. Diabetol Metab Syndr 2015; 28; 7:28.

39. Guiu B, Loffroy R, Hillon P, Petit JM. Magnetic resonance imaging and spectroscopy for quantification of hepatic steatosis: urgent need for standardization! J Hepatol 2009; 51: 1082-1083.

40. Guiu B, Petit JM, Loffroy R, et al. Quantification of liver fat content: comparison of triple-echo chemical shift gradient- echo imaging and in vivo proton MR spectroscopy. Radiology 2009; 250: 95-102.

41. Lupşor-Platon M, Stefănescu H, Mureșan D, et al. Noninvasive assessment of liver steatosis using ultrasound methods. Med Ultrason 2014; 16: 236-245.

42. Badea R. Ficatul. In: Badea RI, Dudea SM, Mircea PA, Stamatian F. Tratat de Ultrasonografie Clinică. Ed. Medicală, Bucureşti, 2000: 105-176.

43. Mathiesen UL, Franzen LE, Aselius H, et al. Increased liver echogenicity at ultrasound examination reflects degree of steatosis but not of fibrosis in asymptomatic patients with mild/moderate abnormalities of liver transaminases. Dig Liver Dis 2002; 34: 516-522.

44. Joy D, Thava VR, Scott BB. Diagnosis of fatty liver disease: is biopsy necessary? Eur J Gastroenterol Hepatol 2003; 15: 539-543.

45. Saadeh S, Younossi ZM, Remer EM, et al. The utility of radiological imaging in nonalcoholic fatty liver disease. Gastroenterology 2002; 123: 745-750.

46. Ozhan H, Alemdar R, Caglar O, et al; MELEN Investigators. Performance of bioelectrical impedance analysis in the diagnosis of metabolic syndrome. J Investig Med 2012; 60: 587-591.

47. Völgyi E, Tylavsky FA, Lyytikäinen A, Suominen H, Alén M, Cheng S. Assessing body composition with DXA and bioimpedance: effects of obesity, physical activity, and age. Obesity (Silver Spring) 2008; 16: 700-705.

48. Pérez-Matute P, Pérez-Martínez L, Blanco JR, et al. Multiple frequency bioimpedance is an adequate tool to assess total and regional fat mass in HIV-positive patients but not to diagnose HIV-associated lipoatrophy: a pilot study. J Int AIDS Soc 2013; 16: 18609.

49. Karason K, Wikstrand J, Sjostrom L, Wendelhag I. Weight loss and progression of early atherosclerosis in the carotid artery: a four-year controlled study of obese subjects. Int J Obes Relat Metab Disord 1999; 23: 948-956.

50. Mavri A, Stegnar M, Sentocnik JT, Videcnik V. Impact of weight reduction on early carotid atherosclerosis in obese premenopausal women. Obes Res 2001; 9: 511-516. 